\title{
Semiconductor Quantum Well Lasers with a Temperature Insensitive Threshold Current
}

\author{
A. R. Adams ${ }^{1}$, I. P. Marko ${ }^{1}$, J. Mukherjee ${ }^{1}$, S. J. Sweeney ${ }^{1}$, A. Gocalinska ${ }^{2}$, E. Pelucchi $^{2}$ and \\ B. Corbett ${ }^{2}$ \\ ${ }^{1}$ Advanced Technology Institute and Department of Physics, University of Surrey, Guildford \\ GU2 $7 X H, U K$ \\ ${ }^{2}$ Tyndall National Inst., University of Cork, Lee Maltings, Dyke Parade, Cork, Ireland \\ alf.adams@surrey.ac.uk
}

Quantum well lasers have been extremely successful in a wide range of applications, with optical fibre communications being of particular importance. However, in spite of their success, their performance is not ideal, for example, the threshold current of semiconductor lasers is often very sensitive to temperature. This has led to the need for thermoelectric coolers and associated control electronics to stabilize the laser temperature, however, such coolers often consume more energy than the laser they are controlling and also add to the overall heat dissipation of the system. Such coolers also tend to have far less long-term reliability than the laser diode itself. There are consequently many circumstances where it would be advantageous and far cheaper to simply compensate for temperature variations by mechanisms built into the epitaxial structure of the laser chip itself. This paper focuses on a new design and demonstration of a MQW laser structure which can overcome the intrinsic temperature sensitivity of the laser.

There are several mechanisms that may cause the threshold current $\left(\mathrm{I}_{\mathrm{th}}\right)$ of semiconductor lasers to increase rapidly with increasing temperature, $T$. These include thermal broadening of the gain spectrum, thermally activated carrier escape, Auger recombination and inter-valence band absorption. Normally all of these processes act together to cause $\mathrm{I}_{\text {th }}$ to increase as $T$ increases. However, here we present a novel design of quantum well active region [1] such that carriers released from some of the wells are fed to the other wells in such a way that these mechanisms compensate rather than augment one another.

The idea is in principle applicable to a range of materials systems, structures and operating wavelengths. However, we chose to demonstrate the effect for the first time in $1.5 \mu \mathrm{m}$ GaInAsP/InP edge emitting lasers, since these have been so extensively characterised and their parameters are relatively well known [2].

The devices were grown by MOVPE with seven $6 \mathrm{~nm}$ wide InGaAsP quantum wells designed using computer simulation. The compressive strain in the wells was compensated overall by tensile strain in the barriers. Devices were fabricated with $50 \mu \mathrm{m}$ and $80 \mu \mathrm{m}$ wide stripe contacts so that any temperature dependent current spreading was negligible. One millimetre long devices were produced with as-cleaved facets.

The results are shown in Fig 1. Sample "A" was the first to be grown and as can be seen, it displayed a constant threshold current over a range of approximately $70 \mathrm{~K}$ but centred at low temperature $(80 \mathrm{~K} \sim 150 \mathrm{~K})$. The device lased at $1.63 \mu \mathrm{m}$ at room temperature and analysis of the emission spectra and TEM of the structure showed that the growth was far from the design. 
However, the fact that a region of constant $\mathrm{I}_{\text {th }}$ existed indicated that the concept was a robust one. Sample "B" was the second to be grown and as can be seen the flat $\mathrm{I}_{\text {th }}$ region was successfully moved from low temperature and now spanned from approximately $140 \mathrm{~K}$ to $270 \mathrm{~K}$. This device lased at $1.47 \mu \mathrm{m}$ at room temperature indicating that the structure was still not exactly as designed. However, the changes made between sample " $\mathrm{A}$ " and sample " $\mathrm{B}$ " were well within the limits of the variations available and therefore provides confidence that further iterations would make it possible to produce a device able to work at, for example, $1.55 \mu \mathrm{m}$ and with a constant $\mathrm{I}_{\text {th }}$ at room temperature and above. This work is in progress and will be presented at the conference.

This generic design approach, which will be presented in more detail in the talk, is equally applicable to $1.3 \mu \mathrm{m}$ lasers as well as shorter wavelengths and to Mid IR devices in both edge-emitting and vertical cavity geometries.

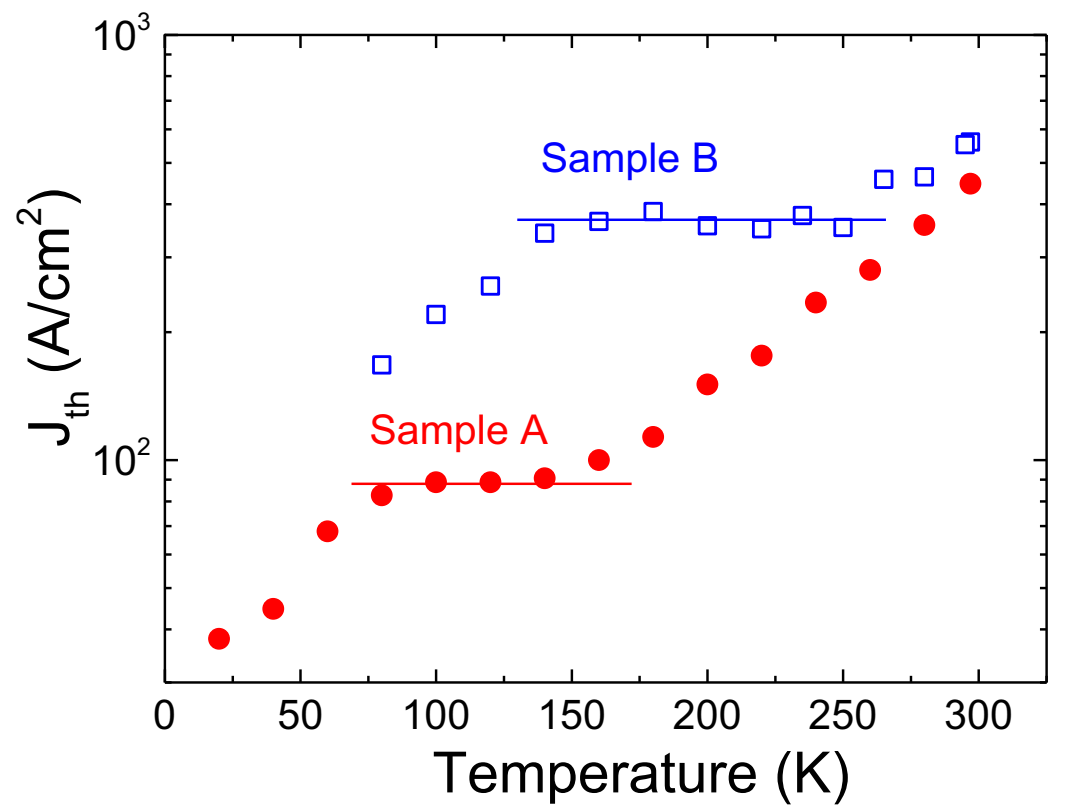

Fig. 1. Temperature dependence of threshold current density in two InGaAsP/InP laser structures grown according to a simulated design with predicted temperature insensitive behaviour.

The authors wish to thank Dr V Stolojan for TEM studies of these structures

\section{References}

[1] A. R. Adams, International Patent Application WO2012/153136 (2012).

[2] A. F. Phillips, S. J. Sweeney, A. R. Adams, P. J. A. Thijs, "The Temperature Dependence of 1.3- and 1.5- $\mu \mathrm{m}$ Compressively Strained InGaAs(P) MQW Semiconductor Lasers", IEEE J. Select. Topics Quant. Electr., 5, iss.3, 401 (1999). 